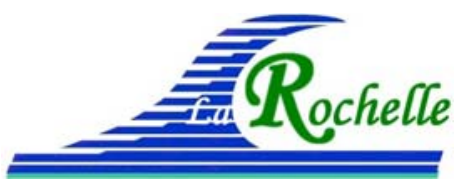

XVèmes Journées Nationales Génie Côtier - Génie Civil

La Rochelle, 29 au 31 mai 2018

DOI:10.5150/jngcgc.2018.076

(C) Editions Paralia CFL

disponible en ligne - $h$ ttp://www.paralia.fr - available online

\title{
Suivi de la turbidité par satellite : un nouvel outil d'aide à la gestion opérationnelle en zone fluvio-estuarienne et littorale
}

\author{
Virginie LAFON ${ }^{1}$, Olivier REGNIERS ${ }^{1}$, Cécile CURTI ${ }^{1}$, Aurélie DEHOUCK ${ }^{1}$
}

1. I-SEA, 25 avenue Marcel Issartier, 33700 Mérignac, France; E-mail:

virginie.lafon@i-sea.fr

\section{Résumé :}

En domaine littoral, l'utilisation des données de télédétection spatiale répond à des besoins de gestion opérationnelle de plus en plus diversifiés. Notre article se focalise sur 3 usages : la gestion des étiages, la gestion des ressources en eau douce, la planification de travaux en mer. Trois études pilotées par des organismes gestionnaires forment le cadre de nos recherches. Les gammes de turbidité inversibles ainsi que les incertitudes sur la mesure par satellite ont tout d'abord été calculées. Puis, la capacité de l'ensemble des constellations de satellites d'observations de la Terre à observer à une fréquence et une échelle spatiale adaptées aux phénomènes d'intérêt suivis a été analysée. Nos résultats montrent que des teneurs comprises entre $10 \mathrm{mg} / \mathrm{L}$ et $6 \mathrm{~g} / \mathrm{L}$ peuvent être mesurées avec une précision de l'ordre de $35 \%$ pour les teneurs supérieures à $100 \mathrm{mg} / \mathrm{L}$. Dans les fleuves tidaux, nous montrons que les données spatiales permettent (1) de mieux comprendre la distribution spatiale des MES, (2) de contribuer à la caractérisation de la dynamique des bouchons vaseux et d'estimer des tendances sur plusieurs décennies, (3) de capitaliser des cas de figure de validation pour les modèles hydro-sédimentaires. Sur le littoral, le suivi par satellite permet (1) d'analyser à grande échelle spatiale la dynamique des sédiments fins, (2) d'analyser le bruit de fond de turbidité, (3) d'aider à anticiper les périodes et zones de travaux à favoriser pour en limiter les impacts. Nous avons également pu montrer que certains phénomènes ne peuvent être systématiquement anticipés, comme l'effet des manœuvres d'écluses ou de barrages sur la turbidité de l'eau. Ainsi, les données satellitaires peuvent contribuer à prendre des mesures de gestion à l'échelle de grandes masses d'eau (baies, fleuves et estuaires) à moyen terme et permettent donc de développer des services opérationnels pour planifier des opérations de dragage et indirectement contribuer à la gestion des étiages dans le cas des secteurs hyperturbides.

\section{Mots-clés :}

Turbidité, Zone côtière, Télédétection spatiale, Qualité de l'eau, Ressources en eau, Gestion opérationnelle.

\section{Introduction}

Les estuaires et les baies littorales abritent une biodiversité riche et des écosystèmes parmi les plus productifs en biomasse. Ceux-ci, particulièrement sensibles et fragiles, 


\section{Thème 6 - Gestion durable des zones littorales et estuariennes}

sont exposés aux impacts potentiels des développements industrialo-portuaires. Si ces secteurs concentrent de nombreuses problématiques, la connaissance du fonctionnement naturel de ces systèmes dynamiques est une des clés permettant d'établir des stratégies pour préserver la qualité du milieu dont dépendent par ailleurs certaines filières économiques. Le maintien des stocks et de la qualité de l'eau sont actuellement les enjeux parmi les plus cruciaux que les gestionnaires de ces milieux doivent maîtriser. Parmi les multiples paramètres qui sont surveillés, notre étude se focalise sur la turbidité. Dans les exemples développés, la dynamique des sédiments fins est particulièrement ciblée. Traditionnellement, les systèmes de surveillance de la turbidité naturelle reposent sur des sondes multi-paramètres de mesure à haute fréquence. Couplés à ces instruments, les modèles hydro-sédimentaires ont été les tout premiers outils pour obtenir une information spatialisée. La télédétection spatiale est, elle, un moyen concret d'observation et de mesure synoptique. En effet, les satellites optiques permettent d'évaluer la turbidité ou la teneur en matières en suspension (MES) en zone côtière et estuarienne (NECHAD et al., 2010 ; DOGLIOTTI et al., 2015 ; HAN et al., 2016). Ces études basées sur des modèles optiques semi-analytiques font état, suivant les secteurs investigués, de précisions qui peuvent, dans le meilleur des cas avoisiner les $20 \%$ pour la gamme $0-2 \mathrm{~g} / \mathrm{L}$ (NOVOA et al., 2017). Nous proposons ici de compléter ces résultats pour des MES plus élevées. En outre, nous montrerons dans quelle mesure les observations satellitaires sont obtenues avec une précision et une fréquence leur conférant la capacité de contribuer à l'aide à la décision dans trois domaines : la gestion des étiages, la gestion des ressources en eau douce, la planification de travaux en mer.

\section{Contexte et localisation des sites d'étude}

Trois sites distincts, macrotidaux et caractérisés par une sédimentation fine, sont abordés dans cette étude : la Garonne et la Dordogne tidales, La Charente aval et les pertuis charentais (figure 1). Pour la Garonne et la Dordogne, nous considérons les sections fluviales soumises à la marée, soit, sur chacun des deux fleuves, un linéaire d'environ $50 \mathrm{~km}$ en amont de l'estuaire. Ces deux fleuves présentent une zone appelée bouchon vaseux où les concentrations dépassent $5 \mathrm{~g} / \mathrm{L}$ en surface (JALON-ROJAS et al., 2017). Les dépôts de vase dans l'estuaire de la Gironde s'effectuent en grande partie au niveau des chenaux par décantation des sédiments en suspension (BERTHOIS, 1964). Sur les estrans, la sédimentation se fait directement depuis le bouchon vaseux (ALLEN et al., 1974). Ce dernier a donc un impact majeur sur l'effort de dragage et sur les écosystèmes (passage des poissons migrateurs, colmatage des frayères). En complément des analyses menées à partir des acquisitions du réseau MAGEST (JALON-ROJAS et al., 2017), à la demande de l'Agence de l'Eau Adour Garonne (AEAG), nous avons exploré le potentiel des images satellite pour le suivi spatiotemporel du bouchon vaseux et pour soutenir la gestion opérationnelle des étiages. 


\section{XVèmes Journées Nationales Génie Côtier - Génie Civil \\ La Rochelle, 29 au 31 mai 2018}

La Charente aval s'étend entre l'embouchure du fleuve et le barrage de Saint-Savinien, $50 \mathrm{~km}$ en amont. Ce secteur présente un bouchon vaseux et de larges estrans vaseux intertidaux. Les concentrations en MES peuvent y atteindre $10 \mathrm{~g} / \mathrm{L}$ en surface (RAVAIL et al., 1998 ; COULOMBIER et al., 2014). A la demande du département de la Charente Maritime, nous nous sommes focalisés sur deux objectifs : l'amélioration à partir des images satellite des connaissances sur la dynamique du bouchon vaseux, et la définition des conditions de risque de remontée du bouchon vaseux en amont des ouvrages des Saint Savinien, vers des prises d'eau potable.

Notre dernier site (pertuis breton et d'Antioche) concerne le secteur compris entre les baies de l'Aiguillon et de Marennes Oléron. Le Port Atlantique La Rochelle, au cœur de ce secteur, entreprend régulièrement des chantiers de déroctage. Afin d'en limiter au maximum les impacts, le Port nous a chargé d'évaluer la capacité des outils de télédétection pour anticiper et contribuer à contrôler les phases de chantier.

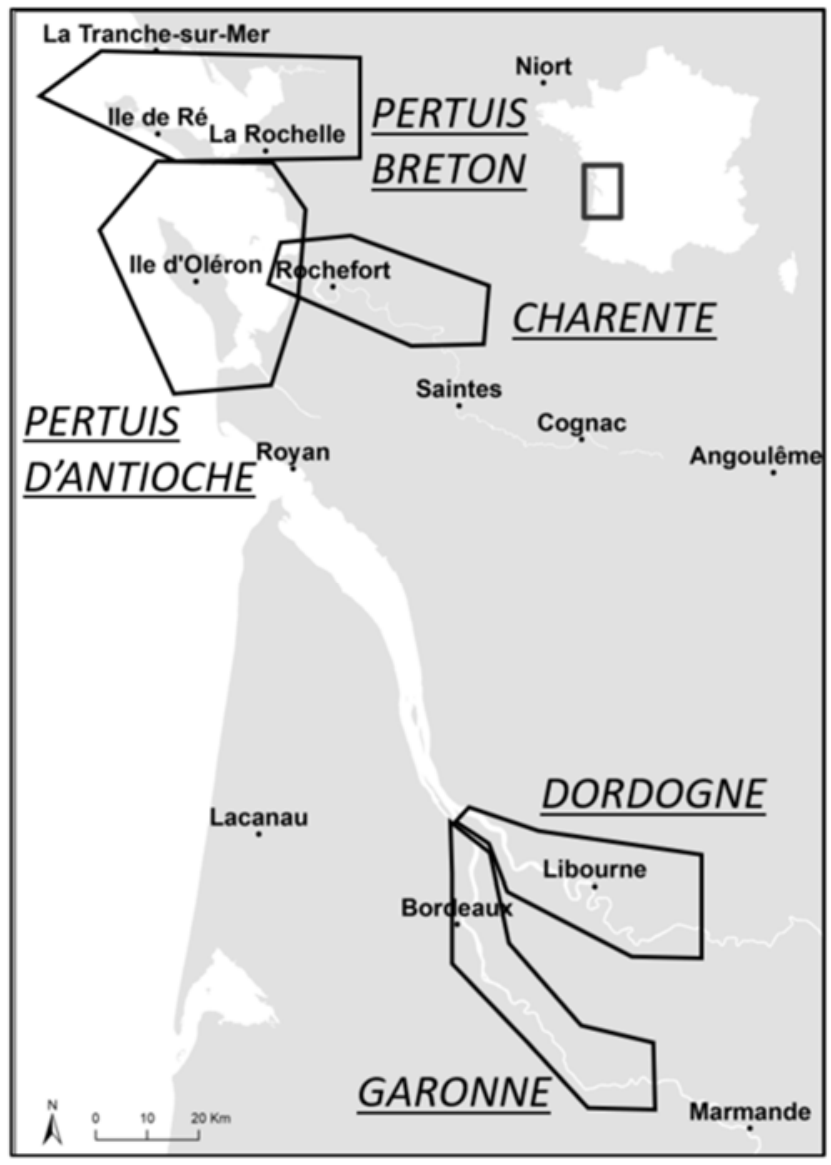

Figure 1. Localisation des sites d'étude.

\section{Méthode et données}

La résolution spatiale et la fréquence d'acquisition de données exploitables (et non la fréquence théorique d'acquisition) caractérisent les capacités du spatial pour suivre la 


\section{Thème 6 - Gestion durable des zones littorales et estuariennes}

dynamique sédimentaire. L'accès aux images, le délai entre acquisition et production de cartes de MES et la période couverte par l'archive en ligne s'ajoutent à ces caractéristiques pour conditionner les usages du spatial.

\subsection{Images satellites}

A haute résolution spatiale (HR), nous avons exploré les archives Landsat et Sentinel-2 (S2). Landsat-8, lancé en 2013, a une capacité de revisite en chaque point du globe de 16 jours et une résolution spatiale de $30 \mathrm{~m}$ dans les bandes visibles et proche infrarouge (VNIR) utiles pour caractériser les MES. Les premières images Landsat datent du début des années 70. Depuis 1999, les prises de vue sont systématiques, archivées et distribuées gratuitement (Earth Explorer, USGS). S2-A et S2-B ont été lancés respectivement en 2015 et 2017 dans le cadre du programme Copernicus de l'Agence Spatiale Européenne (ESA). Les images S2 ont une résolution de $10 \mathrm{~m}$ dans les bandes VNIR. Leur capacité combinée de revisite d'un même site est de 5 jours. Les acquisitions S2 sont systématiques, archivées et téléchargeables gratuitement (SciHub, ESA). A moyenne résolution spatiale (MR), nous avons exploité les données VIIRS, mis en orbite fin 2011. Les images VIIRS brutes contiennent 9 bandes spectrales dans les longueurs d'onde VNIR avec une résolution au nadir de $750 \mathrm{~m}$. La revisite en tout point du globe est de 1 à 2 fois par jour.

Les images brutes sont corrigées des effets atmosphériques pour appliquer les lois d'inversion de la réflectance en MES. Des produits corrigés en réflectance terrestres sont fournis par l'USGS (Landsat) et le CNES (Sentinel). Ils sont adaptés à l'inversion de la turbidité par des algorithmes empiriques (LAFON et al., 2016). Les images en réflectances marines calculées par le logiciel ACOLITE (VANHELLEMONT \& RUDDICK, 2016) pour Landsat et S2 et fournies l'Ocean Biology Processing Group (OBPG) de la NASA pour VIIRS (pixels VIIRS ré-échantillonnés à $1 \mathrm{~km}$ ) sont exploitées par les méthodes d'inversion semi-analytiques.

\subsection{Données in situ}

Chacun des sites d'étude a fait l'objet de campagnes terrain ayant pour double objectif d'analyser la sensibilité des capteurs optiques à la teneur en MES et d'étalonner des lois empiriques d'inversion entre couleur de l'eau et turbidité, et ce pour tous les satellites ciblés. Les algorithmes définis sont sites spécifiques. Sur le terrain, sont collectés simultanément la réflectance de l'eau au moyen de spectroradiomètres et un prélèvement d'eau analysé ensuite en laboratoire afin d'estimer les MES. Les spectres acquis sont traités pour simuler les réflectances des bandes spectrales VNIR de chaque capteur. Ces réflectances simulées sont mises en relation avec les MES associées afin d'analyser la sensibilité spectrale des capteurs aux gammes de MES observées et d'établir des modèles d'inversion en choisissant la/les bande(s) spectrale(s) adéquate(s). La gamme de MES observée lors de ces campagnes s'étend de $15 \mathrm{mg} / \mathrm{L}$ à 15,5 g/L. 


\section{XVèmes Journées Nationales Génie Côtier - Génie Civil \\ La Rochelle, 29 au 31 mai 2018}

D'autre part, des observations (MES ou NTU) ont été collectées pour valider les MES satellite (données matchups). Au total, nous avons pu réunir 145 matchups sur la Garonne et la Dordogne (MAGEST, SCHMIDT et al., 2016 et données de l'étude AEAG), 16 sur la Charente (données université de La Rochelle) et 26 dans la zone des pertuis (données CREOCEAN). Ces couples de données couvrent la gamme $2 \mathrm{mg} / \mathrm{L} \mathrm{-}$ 4,9 g/L. Ces données nous permettent de compléter les résultats de NOVOA et al. (2017) tout en comparant modèles semi-analytiques et empiriques.

\subsection{Données de forçage}

Nous avons collecté, pour ces études, les données de débit de la base de données HYDRO du MEDDE (http://www.hydro.eaufrance.fr/), les prédictions de marée du SHOM (http://maree.shom.fr/) et les paramètres de vent et houle du modèle WaveWatchIII de la NOAA (http://polar.ncep.noaa.gov/waves/wavewatch/).

\section{Résultats et discussion}

\subsection{Performances et limites d'application des algorithmes d'inversion}

Les campagnes terrain confirment que la sensibilité radiométrique varie avec la gamme de MES considérée. Les faibles turbidités $(<\sim 10 \mathrm{mg} / \mathrm{L})$ se corrèlent bien avec la bande spectrale verte. Les turbidités moyennes $(\sim 10 \mathrm{mg} / \mathrm{L}<\mathrm{MES}<\sim 100 \mathrm{mg} / \mathrm{L})$ sont mieux corrélées avec bande rouge quand, pour des MES $>\sim 100 \mathrm{mg} / \mathrm{L}$ la meilleure corrélation est obtenue avec le rapport de bandes proche infrarouge sur vert. La gamme de MES à laquelle les capteurs optiques est sensible est comprise entre $2 \mathrm{mg} / \mathrm{L}$ et $6 \mathrm{~g} / \mathrm{L}$ (figure 2). Concernant les performances, nous n'arrivons pas à reproduire les scores de NOVOA et al. (2017). Les auteurs présentent des résultats pour un nombre très réduit de points de contrôle, extraits de 10 images Landsat, sélectionnées de manière à atténuer les biais que la comparaison entre des mesures satellite et terrain, rarement synchronisées et distantes de plusieurs mètres, peuvent induire. Notre jeu de données est plus important, plus complet mais aussi moins sélectif. Nous montrons, dans tous les cas, que les lois d'inversion calibrées in situ, et validées avec un jeu de données indépendant, sont toujours plus performantes que les modèles semi-analytiques que nous avons testés (NECHAD et al., 2010; DOGLIOTTI et al., 2015). Ces modèles reposent sur l'inversion de réflectances marines, qui semblent mal restituées par ACOLITE dans les sections des fleuves les plus étroites. Les erreurs d'estimation y sont, en effet, beaucoup plus élevées $(>60 \%)$.

Pour la gamme 2 - $100 \mathrm{mg} / \mathrm{L}$, les erreurs d'estimation des modèles empiriques appliqués aux images HR (Landsat et S2) sont de l'ordre de 40\%, les MES satellite étant globalement sous-estimées par rapport aux MES terrain (figure 3). Au-delà de 100 $\mathrm{mg} / \mathrm{L}$, les MES sont estimées à $35 \%$ près en moyenne. Entre 1 et $5 \mathrm{~g} / \mathrm{L}$, la barre d'erreur sur la mesure semble augmenter graduellement, les plus fortes incertitudes apparaissent 
entre 3,5 et $5 \mathrm{~g} / \mathrm{L}$ (figure 3). Les turbidités VIIRS (26 matchups), montrent une barre d'erreur de $40 \%$ pour la gamme 10-50 NTU (environ 10 à $50 \mathrm{mg} / \mathrm{L}$ ).

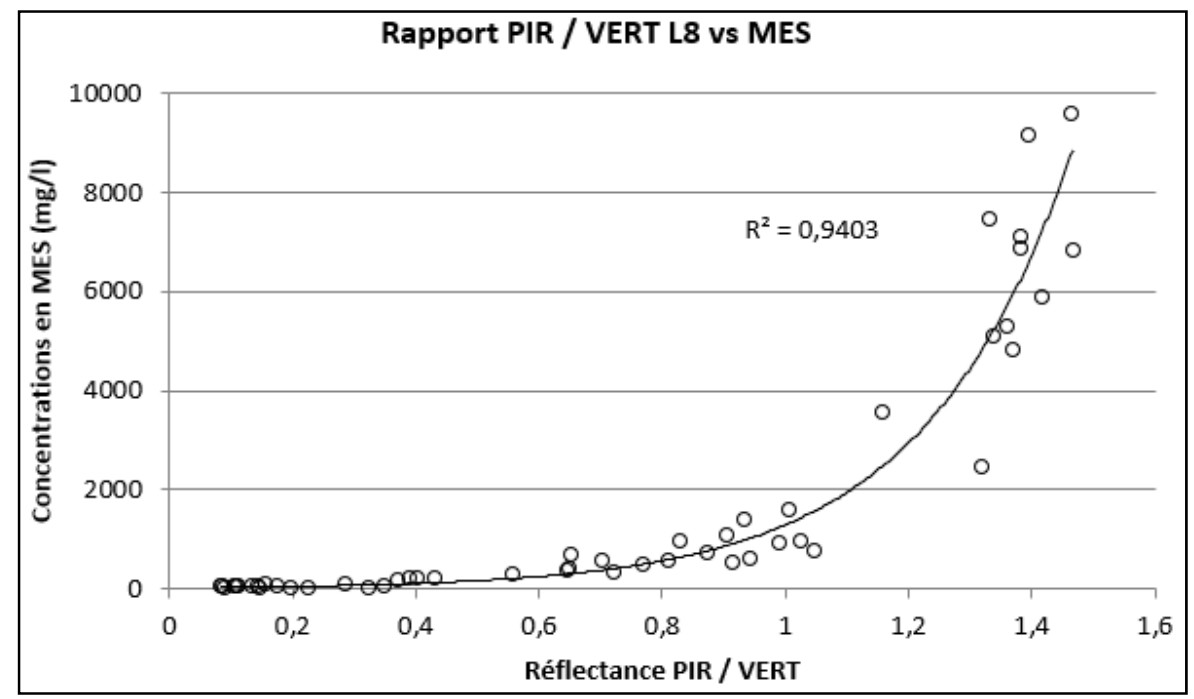

Figure 2. Sensibilité du rapport des bandes spectrales proche infrarouge (PIR) sur vert à la teneur en MES (exemple sur la Charente pour le capteur Landsat 8).

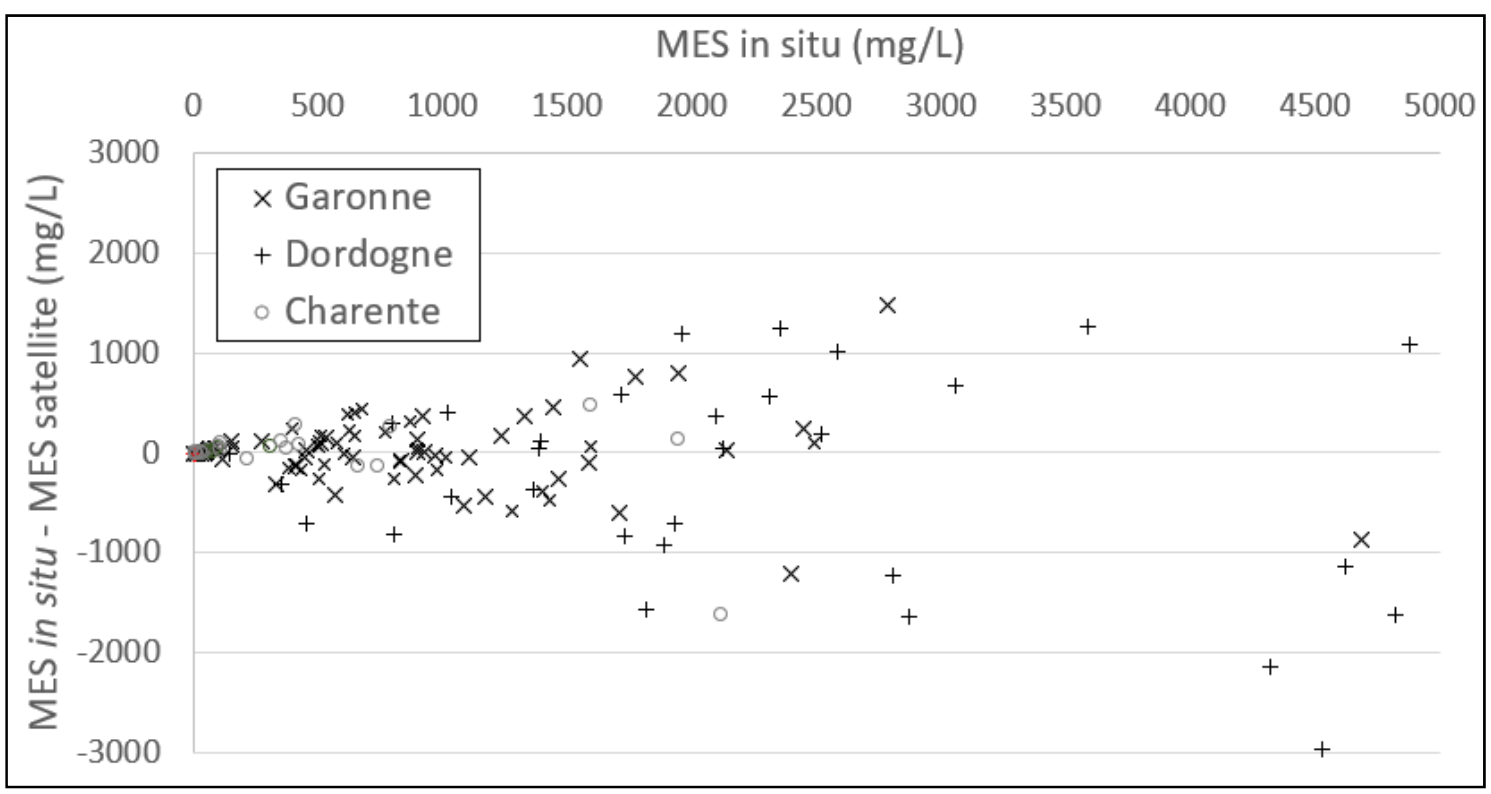

Figure 3. Comparaison MES satellite - MES in situ.

Les ordres de grandeurs et gradients de MES peuvent donc être très correctement appréciés par satellite. En revanche, de telles barres d'erreur ne permettent pas d'établir des seuils d'alerte fins, s'ils sont nécessaires pour préserver des espèces peu tolérantes. 


\section{XVèmes Journées Nationales Génie Côtier - Génie Civil \\ La Rochelle, 29 au 31 mai 2018}

Il faut enfin souligner que les teneurs estimées ne représentent que les premiers $\mathrm{cm}$ de la colonne d'eau. Les cartes produites ne permettent pas de calculer des volumes de MES.

\subsection{Capacités et limites de la télédétection spatiale pour le suivi des turbidités}

Les archives HR remontent au début des années 70 . Les prises de vue sont opportunistes jusqu'à la fin des années 90 . C'est également à partir de cette période que se construit l'archive image MR. Suivant la régularité des acquisitions vis-à-vis des processus suivis, des tendances peuvent être analysées avec un recul de 20 voire 40 à 50 ans.

Aujourd'hui, quel que soit le capteur, les produits MES peuvent être fournis dans les 48h après l'acquisition.

Les capteurs HR permettent d'étudier des sections de fleuves et estuaires de plus en plus étroites. Entre 1972 et 1984, seules les sections supérieures à $250 \mathrm{~m}$ étaient discernables. Depuis 1984, on peut étudier des secteurs où les rives seront distantes d'au moins $100 \mathrm{~m} ; 50 \mathrm{~m}$ depuis 2015 . Au débouché des estuaires les plus vastes ainsi que dans les baies, les enjeux liés à la résolution spatiale des capteurs sont moindres. Toutefois, il faut souligner que les pixels les plus proches de la côte sont toujours masqués. VIIRS, et plus généralement tous les produits en réflectance marine de l'OBPG, ne permet pas d'obtenir des observations à moins d'un km du littoral. Ainsi, suivant les secteurs visés par l'analyse, les données VIIRS ne seront pas éligibles.

VIIRS, à l'instar de tous les capteurs MR (ex. MODIS, Sentinel-3), a une fréquence d'observation élevée. Plusieurs images par jour sont acquises. Elles sont exploitables s'il n'y a pas de nuages. Les images HR sont, elles, beaucoup moins nombreuses. Dans le meilleur des cas, 4 à 5 images par mois sont exploitables l'été (juillet-octobre), on peut en trouver au maximum 2 par mois en moyenne le reste de l'année. Ces fréquences d'acquisition limitent les processus suivis aux variabilités, respectivement, saisonnières à interannuelles pour la HR, et liées au cycle de marée à interannuelles pour la MR.

\subsection{Capacité de la télédétection spatiale pour des usages opérationnels}

\section{Gestion des étiages et des ressources en eau}

Les observations satellitaires ne sont pas exploitables pour l'aide à la gestion lorsque la réactivité attendue est inférieure à $48 \mathrm{~h}$. C'est par exemple le cas pour manœuvrer les vannes de pompage pour les usines de production d'eau potable et éviter l'entrée de MES dans le circuit de filtration. En outre, la sensibilité de ces circuits impose une quantification fine des teneurs en MES qui n'est pas toujours compatible avec les barres d'erreur de la mesure par satellite.

En revanche, les séries d'images HR acquises ces 20 dernières années sont utiles pour améliorer la compréhension de la dynamique des bouchons vaseux fluvio-estuariens. Les images produites montrent qu'un fort gradient de MES marque la limite amont du bouchon vaseux. En comparant des observations terrain et image (données AEAG), 


\section{Thème 6 - Gestion durable des zones littorales et estuariennes}

nous pouvons préciser que cet indicateur permet d'estimer la position des limites amont d'intrusion du bouchon vaseux à 2 à $5 \mathrm{~km}$ près. Les dates encadrant la période de migration vers l'amont des bouchons vaseux peuvent être estimées à 2 voire 6 semaines près. Cette approximation est liée à la relativement faible fréquence d'obtention d'images exploitables qui, de plus, n'offrent qu'une représentation partielle des conditions de forçage naturelles même en ne considérant que le débit et la marée. De ce fait, une analyse prudente de ces données doit être menée pour évaluer des tendances évolutives, même à l'échelle interannuelle. Les études que nous avons menées ne nous ont pas permis de définir d'indicateurs pertinents, basées sur les MES pour l'aide à la gestion des périodes d'étiage.

\section{Planification de travaux en mer}

Pour la planification des travaux en mer, la connaissance de la turbidité naturelle est utile pour contribuer au choix des seuils d'alerte pour réguler les opérations de rejet des eaux de dragage. Afin d'analyser le bruit de fond de turbidité dans la zone des pertuis, nous avons téléchargé trois années de données et VIIRS. Au total 200 images se sont révélées exploitables. En croisant cette base de données aux données de forçage, nous montrons que : (i) la diversité des coefficients de marée est correctement représentée, quelle que soit la saison considérée (à gauche, figure 4), (ii) la représentativité des moments de marée est hétérogène, le jusant étant largement sous-représenté (à droite, figure 4), (iii) les périodes de fort vent, moins fréquemment représentées couvrent toutefois toute la gamme de coefficients de marée, enfin les directions des vents dominants (KERVELLA, 2009) sont globalement correctement représentées à l'échelle annuelle, (iv) les conditions de vagues connues (KERVELLA, 2009) sont bien représentées.

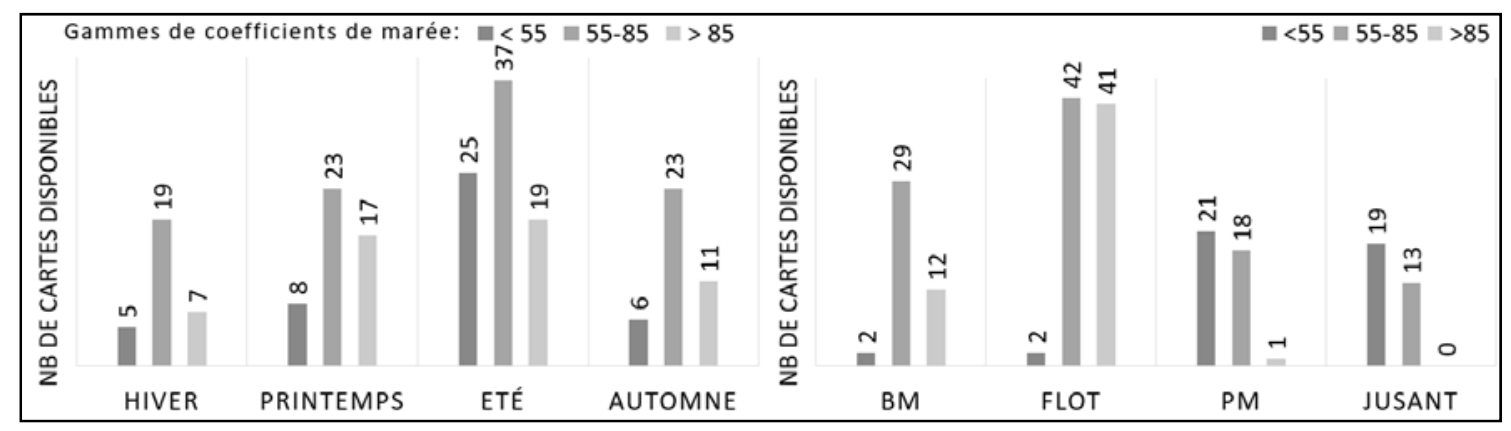

Figure 4. Nombre d'images disponibles (pertuis charentais) classées suivant le coefficient de marée et la saison (gauche), le coefficient et le moment de marée (droite)

Les conclusions de l'analyse croisée forçage / images ne peuvent être détaillés ici. Elles montrent globalement que les 200 observations permettent de discriminer 9, dont 5 décrire le fonctionnement du pertuis Breton (figure 5) cas de figure emblématiques de la 


\section{XVèmes Journées Nationales Génie Côtier - Génie Civil La Rochelle, 29 au 31 mai 2018}

distribution des MES dans les eaux de surface dépendantes des conditions de forçage dominantes. Les niveaux de MES qui leur sont associés sont également définis. La cohérence et l'exhaustivité des résultats obtenus, confrontés à l'analyse de sondes installées plusieurs mois sur le terrain (données CREOCEAN), nous permettent de faire l'hypothèse que la moindre représentativité des conditions de jusant ne biaise pas significativement les résultats de notre analyse. La série de cartes produites peut également être exploitée pour définir des zones distantes dans les deux baies où la turbidité naturelle varie de façon synchrone et dans les mêmes ordres de grandeur et ainsi sélectionner des zones de référence pour contrôler ensuite en temps réel et sur le terrain l'impact de chantier en mer.

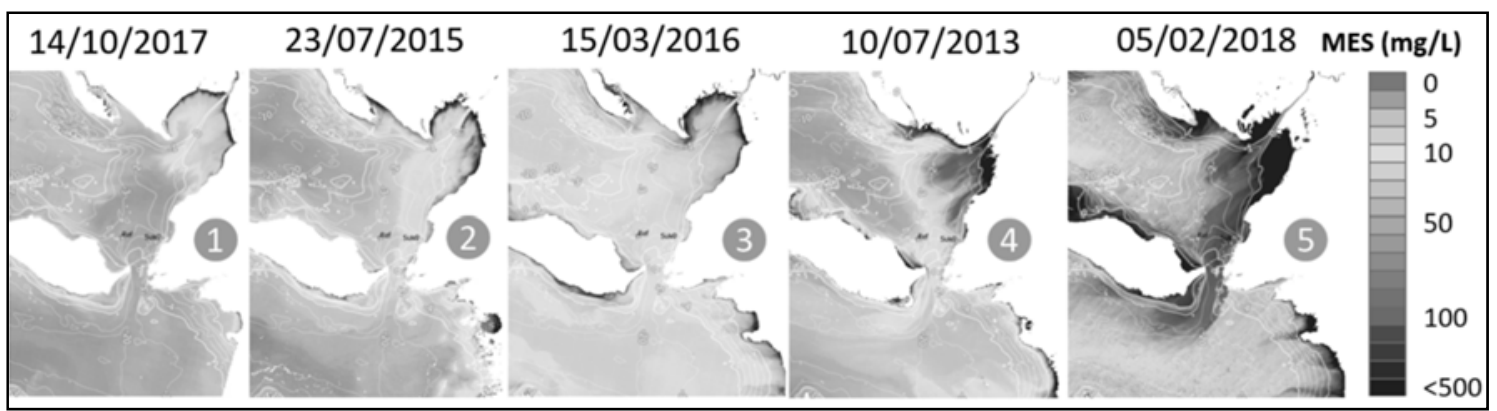

Figure 5. Cas de figures emblématiques de la distribution spatiale des MES sans le pertuis Breton (données Landsat et S2)

\section{Conclusions}

Nous montrons, au travers de cas de figure concrets, que les séries temporelles d'images satellitaires sont utiles pour accroître la connaissance de la dynamique sédimentaire littorale et contribuer dans certains cas à la gestion du milieu. Les MES sont détectées sur une vaste gamme (2 à $6000 \mathrm{mg} / \mathrm{L}$ ) avec des précisions comprises entre 20 et $40 \%$ au mieux. Les données MR sont performantes pour analyser à grande échelle la turbidité naturelle d'un site, et pour en caractériser un nombre significatif d'aspects en fonction des conditions de forçage, y compris l'effet des tempêtes et du coefficient de marée. Ces données peuvent efficacement contribuer à définir les conditions et protocoles de suivi des chantiers en mer, mais ne sont pas adaptées au suivi lui-même, en temps réel. Dans les fleuves et estuaires le données HR sont exploitées. Relativement peu nombreuses à l'échelle annuelle, elles sont adaptées au suivi intra-saisonnier (en période d'étiage) à interannuel. Elles peuvent aider à surveiller des tendances évolutives (ex. localisation du bouchon vaseux) sur plusieurs décennies. Les études basées sur ces bases de données doivent toujours démontrer l'exhaustivité des conditions de forçages naturelles représentées base de données, même pour les bases de données MR. 


\section{Thème 6 - Gestion durable des zones littorales et estuariennes}

\section{Références bibliographiques}

ALLEN G. P., BONNEFILlE R., COURTOIS G, MIGNIOT C. (1974). Processus de sédimentation des vases dans l'estuaire de la Gironde. Contribution d'un traceur radioactif pour l'étude du déplacement des vases. La Houille Blanche, Vol. 1/2, pp 129136. https://doi.org/10.1051//hb/1974013

BERTHOIS L. (1964). Etudes des dynamiques estuariennes dans le fleuve "La Loire". 4eme Congrès International Portuaire, Anvers, 22-27 juin 1964, section Ill, 1re partie, pp 32-41.

COULOMBIER T., TOUBLANC F., BRENON I. (2014). Seasonal monitoring of sediment dynamics in a highly turbid estuary (Charente estuary, France): source and sink of the turbidity maximum. Coastal Dynamics, pp 387- 396.

DOGLIOTTI A., RUDDICK K., NECHAD B., DOXARAN D., KNAEPS E. (2015). A single algorithm to retrieve turbidity from remotely-sensed data in all coastal and estuarine waters. Remote Sensing of Environment, Vol. 156, pp 157-168. https://doi.org/10.1016/j.rse.2014.09.020

JALON-ROJAS I., SCHMIDT S., SOTTOLICHIO A. (2017). Comparison of environmental forcings affecting suspended sediments dynamics in two macrotidal, highly-turbid estuaries. Estuarine and Coastal Shelf Science, Vol. 198, pp 529-541. https://doi.org/10.1016/j.ecss.2017.02.017

KERVELLA S., 2009. Dynamique des sédiments fins et mixtes des zones intertidales de la baie de Marennes-Oléron. Thèse de Doctorat de l'Université de La Rochelle, $310 \mathrm{p}$.

LAFON V., MULAMBA-GUILHEMAT E., GERNEZ P., DOXARAN D., VERNEY R., CURTI C., LUBAC B., SOTTOLICHIO A., DEHOUCK A., REGNIERS O. (2016). Monitoring turbidity changes in estuaries using Sentinel-2 \& -3 constellations. Living Planet Symposium, ESA, 9-13 May 2016, Prague.

NECHAD B., RUDDICK K. G., PARK Y. (2010). Calibration and validation of a generic multisensor algorithm for mapping of total suspended matter in turbid waters.

Remote Sensing of the Environment, Vol. 114, pp 854-866. https://doi.org/10.1016/j.rse.2009.11.022

RAVAIL B., HERAL M., MAESTRINI S., ROBERT J.-M. (1988). Incidence du débit de la Charente sur la capacité biotique du bassin ostréicole de Marennes-Oléron. Journal de Recherche Océanographique, Vol. 13, pp 48-52.

SCHMIDT S., ETCHERBER H., SOTTOLICHIO A., CASTAING P. (2016). Le réseau MAGEST : bilan de 10 ans de suivi haute-fréquence de la qualité des eaux de l'estuaire de la Gironde. Mesures haute résolution dans l'environnement marin côtier, Edts Schmitt F., \& Lefevre A., Presses du CNRS.

VANHELLEMONT Q., RUDDICK K. (2016). Acolite for Sentinel-2: Aquatic applications of MSI imagery. In Proc. the 2016 ESA Living Planet Symposium, ESA Special Publication SP-740. 